\title{
X-ray observations of central stars of planetary nebulae and their winds
}

\author{
Martín A. Guerrero \\ Instituto de Astrofísica de Andalucía (IAA-CSIC), Granada, Spain \\ email: mar@iaa.es
}

\begin{abstract}
The photospheric emission from the hottest central stars of planetary nebulae (CSPNe) is capable to extend into the X-ray domain, with emission peaking at $0.1-0.2 \mathrm{keV}$ and vanishing above $0.4 \mathrm{keV}$. Unexpected, intriguing hard $\mathrm{X}$-ray emission with energies greater than $0.5 \mathrm{keV}$ has been reported for several CSPNe and for a number of white dwarfs (WDs). Different mechanisms may be responsible for the hard X-ray emission from CSPNe and WDs: coronal emission from a late-type companion, shocks in fast winds as in OB stars, leakage from underneath the star photosphere, or accretion of material from a disk, a companion star, or the circumstellar medium. Therefore, the hard X-ray emission associated with CSPNe may have significant implications for our understanding of the formation of PNe: binary companions, disks, and magnetic fields are thought to play a major role in the shaping of $\mathrm{PNe}$, whereas clumping in the stellar wind may have notable effects in the PN evolution by modifying the stellar mechanical energy output. Here I present the results of different observational efforts to search for hard X-ray emission from CSPNe and discuss the different mechanisms for the production of hard X-rays.
\end{abstract}

Keywords. planetary nebulae: general, X-rays: ISM

\section{Introduction}

The central stars of planetary nebulae (CSPNe) are expected to harbor two sources of X-ray emission. First, the photospheric emission from hot $\left(T_{\text {eff }}>100,000 \mathrm{~K}\right)$ CSPNe have high-energy tails that may extend into the X-ray domain. Indeed, recent non-LTE models of CSPN photospheres show further extensions into the X-ray domain than simplistic blackbody models (Rauch2003). This soft photospheric X-ray emission was detected by early X-ray mission in a number of CSPNe: A 36, K 1-16, K 1-27, LoTr 5, NGC 246, NGC 1360, NGC 3587, NGC 4361, NGC 6853, and NGC 7293 (de Korte et al. 1985; Tarafdar \& Apparao 1988; Apparao \& Tarafdar 1989; Kreysing et al. 1992; Rauch, Köppen \& Werner 1994; Hoare et al. 1995). Chandra has recently confirmed the soft X-ray emission from some of these CSPNe (Montez et al. 2012).

A systematic search of the entire ROSAT PSPC archive yield the detection of soft X-ray emission from 13 CSPNe (Guerrero, Chu \& Gruendl 2000) that were point sources at the spatial resolution of $30^{\prime \prime}$ provided by ROSAT PSPC and whose X-ray spectra peaked below $0.2 \mathrm{keV}$ and could be fit using photospheric models. A statistical analysis of the occurrence of soft X-ray emission among CSPNe revealed that ROSAT could only detect sources at small distances and affected by negligible amounts of intervening material, i.e., when detection conditions were ideal. Furthermore, there was a clear trend towards the detection of objects whose nebulae and CSPNe showed indications to be at late evolutionary stages: high $T_{\text {eff }}$ and low photospheric metal content of the CSPN, and large and optically thin nebulae. All these results indicate that the soft photospheric emission from CSPNe is faint and easily absorbed by the intervening material in the nebula and interstellar medium. 

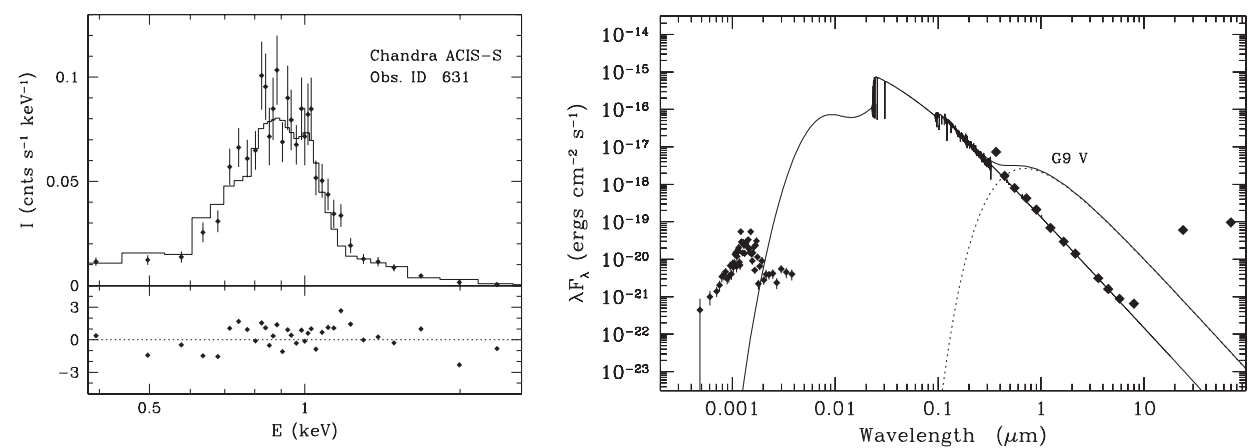

Figure 1. (left) Chandra ACIS-S background-subtracted spectrum of the CSPN of NGC 7293, the Helix Nebula. The spectrum is overplotted by the best-fit thermal plasma emission model with temperatures of $0.65 \mathrm{keV}$ and $1.3 \mathrm{keV}$ and X-ray luminosity $(6.4-7.7) \times 10^{29} \mathrm{erg} \mathrm{s}^{-1}$. The residuals of the fit are shown in the lower panel. (right) Spectral energy distribution of the CSPN of NGC 7293 using Chandra X-ray data, FUSE and IUE UV spectra, ground-based optical and near-IR photometry, and Spitzer IRAC mid-IR photometry. The solid line shows a non-LTE model (Rauch 2003) and the dotted line the contribution from a G9 V dwarf companion.

The X-ray emission from a CSPN may also come from the coronal emission of an unresolved late type companion. There are many examples in the literature of white dwarfs (WDs) with hard X-ray emission that is, indeed, associated with a companion (see O'Dwyer et al. 2003 for a revision of the hard X-ray emission from WDs, and Chu et al. 2004 and Bilíková et al. 2010 for additional extensions). The discovery of hard X-ray emission from a CSPN may thus have significant implications. The evolution of the CSPN through a common envelope phase in a binary system is recognized as the most natural mechanism to produce the most asymmetrical shapes observed among PNe (see the excellent review by de Marco 2009).

Disks, also associated to a close binary, may play a significant role in the launching and collimation of the fast collimated outflows detected in PNe. These can also be responsible for the point-symmetric distribution of jets (e.g., IC 4634; Guerrero et al. 2008) as disks precess or wobble (Livio \& Pringle 1996; Soker 1997). If a disk is present and accretion occurs, then we should expect the production of hard, continuum-dominated X-rays (Soker \& Kastner 2002).

It is necessary to emphasize that X-ray observations of CSPNe can shed light in our understanding of the formation of PNe through the discoveries of binary companions and disks.

\section{Detections of hard X-ray CSPNe}

In the last years, the number of detections of hard X-ray emission in CSPNe has been continuously increasing. Certainly the unprecedented spatial resolution and sensitivity of the Chandra X-ray Observatory is at the root of this series of discoveries, but the history started well before, in the mid 90's when a deep ROSAT PSPC observation detected a source of hard X-ray emission at the central star of the Helix Nebula, NGC 7293 (Leahy, Zhang \& Kwok 1994). The limited spatial resolution of these ROSAT PSPC observations made inconclusive the origin of this hard X-ray emission, but the idea that it originates at the CSPN was supported by the authors. Years later, in 2001, Chandra observations confirmed that the source of hard X-ray emission in NGC 7293 is a point source spatially coincident with its CSPN (Guerrero et al. 2001). The spectrum of this hard X-ray source, peaking at $\sim 0.9 \mathrm{keV}$ (Fig. 1-left), cannot be interpreted as photospheric emission. 


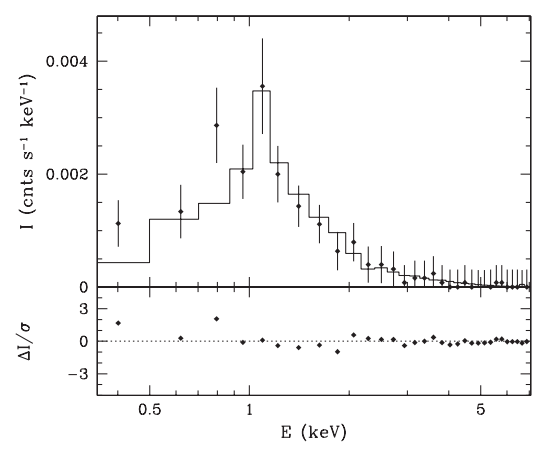

Figure 2. Chandra ACIS-S background-subtracted spectrum of the CSPN of NGC 2392, the Eskimo Nebula. The spectrum is overplotted by the best-fit model of thermal plasma emission with a temperature of $2.8 \mathrm{keV}$ and X-ray luminosity of $3 \times 10^{30} \mathrm{erg} \mathrm{s}^{-1}$. The residuals of the fit are shown in the lower panel.

The ROSAT search for diffuse X-ray emission from PNe suggested that the Cat's Eye Nebula, NGC 6543, was a perfect candidate for the detection of a hot bubble (Guerrero, Chu \& Gruendl 2000). Subsequent Chandra observations resolved the diffuse X-ray emission of the shocked stellar wind within the rim of NGC 6543 (Chu et al. 2001), but they also resulted in the serendipitous discovery of a source of hard X-ray emission at its CSPN (Guerrero et al. 2001). Although the Cat's Eye Nebula show intriguing precessing-like collimated outflows (Miranda \& Solf 1992; Balick 2004) that have been attributed to a companion, the hard X-ray emission from its CSPN was completely unexpected.

Similarly, Chandra observations in the search for diffuse X-ray emission within the central cavities of NGC 2392 and NGC 6826 have produced additional serendipitous discoveries of hard X-ray emission at their CSPNe (Guerrero et al. 2012, in prep.). It is worthwhile to emphasize that the CSPN of NGC 2392 displays the hardest X-ray emission associated to any CSPNe, with a spectrum peaking above $1 \mathrm{keV}$ and a hard energy tail extending up to $5 \mathrm{keV}$ (Fig. 2) that is fitted by an optically thin plasma emission model at a temperature of $2.8 \mathrm{keV}$, i.e., $3.3 \times 10^{7} \mathrm{~K}$.

Whereas previous detections of hard X-ray sources at CSPNe have been fortuitous, there are a series of CSPNe where hard X-ray emission could be expected and, indeed, it has been detected. These are the binary CSPNe at the core of DS 1, HFG 1, and LoTr 5 . The CSPNe of DS 1 and HFG 1 are post common envelope stars consisting of O-type sub-dwarfs with late-type, main-sequence companions (Miszalski et al. 2009), and the CSPN of LoTr 5 is a known binary (may be even ternary) system where the companion is the G5 III variable star IN Com (Jasniewicz, Duquennoy \& Acker 1987). Dedicated Chandra X-ray observations have unveiled hard X-ray emission associated to DS 1 and HFG 1 (Montez et al. 2010). Meanwhile, Chandra and XMM-Newton observations of the nearby X-ray bright group of galaxies RX J1256.0+2556 have serendipitously unveiled the hard X-ray emission of the CSPN of LoTr 5 (Montez et al. 2010; Guerrero et al. 2012, in preparation). Its XMM-Newton spectrum, shown in Figure 3, is indicative of plasma emission at temperatures up to $3.6 \times 10^{7} \mathrm{~K}$.

The number of CSPNe that display hard X-ray emission keeps increasing as more deep and high-resolution X-ray observations are available. An excellent example of this trend is the recent discovery of hard X-ray emission associated with the CSPNe of K 1-16, NGC 1514, and NGC 7009 (Montez et al. 2012) in the framework of the Chandra PN Survey (ChanPLaNS) led by J. Kastner. We should expect more of these discoveries as this survey progresses and obtains more observations of PNe. 

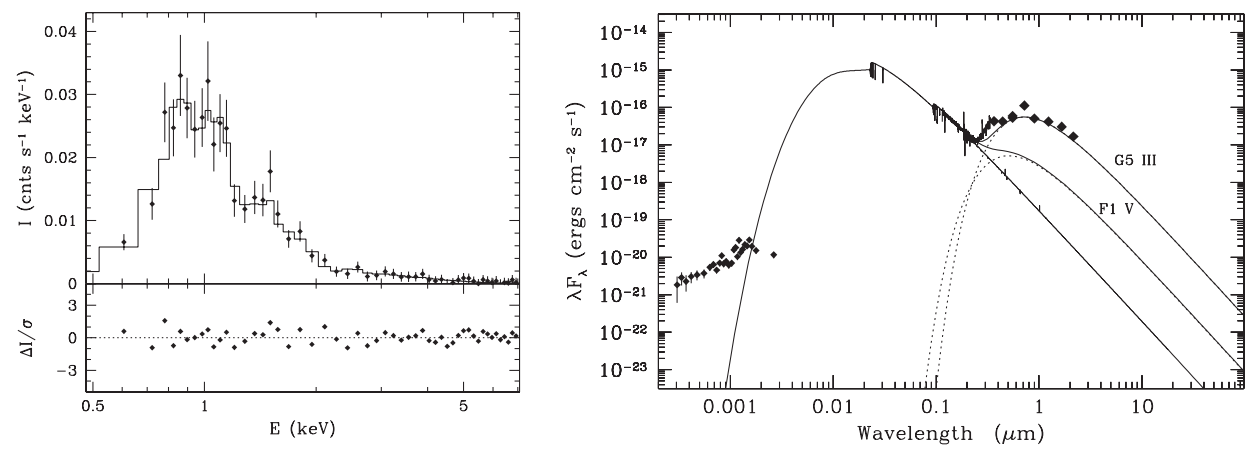

Figure 3. (left) XMM-Newton EPIC-pn spectrum of the CSPN of LoTr 5. The spectrum is overplotted by the best-fit model of thermal plasma emission with temperatures of $0.61 \mathrm{keV}$ and $3.1 \mathrm{keV}$ and X-ray luminosity of $8.0 \times 10^{30} \mathrm{erg} \mathrm{s}^{-1}$. The residuals of the fit are shown in the lower panel. (right) Spectral energy distribution of the CSPN of LoTr 5 using XMM-Newton X-ray data, IUE UV spectra, and ground-based optical and near-IR photometry. The solid line shows a non-LTE model (Rauch 2003) and the dotted line the expected contribution from F1 V or G5 III companions. The match for the G5 III companion is excellent.

\section{Interpretation of hard X-ray emission in CSPNe}

Different mechanisms can be invoked to produce the hard X-ray emission observed in CSPNe. I shall discuss them in the following sections, providing examples of CSPNe whose $\mathrm{X}$-ray emission and general properties fit into these different processes of X-ray emission.

\subsection{Coronal emission from a late companion}

Coronal X-ray emission is characteristic of late stars of spectral types A to M (Hünsch, Schmitt \& Voges 1998; Hünsch et al. 1999). As for the Sun, the X-ray emission is powered by the interplay between stellar rotation and convection in the envelope. The typical value of the $L_{\mathrm{X}} / L_{\mathrm{bol}}$ ratio is $\sim 10^{-5.2}$, with saturated values up to $L_{\mathrm{X}} / L_{\mathrm{bol}} \sim 10^{-3}$ (Güdel 2004).

In late type stars, $L_{\mathrm{X}}$ is related to rotation, $L_{\mathrm{X}} \propto v_{\mathrm{rot}}^{2}$ (Pallavicini et al. 1981). Since the stellar wind will slow down the rotation as the star evolves because the braking action of magnetic field lines carried with the wind, the levels of coronal X-ray emission in late type stars are expected to decline with age. X-ray observations of single late type stars show that this is indeed the case, with $L_{\mathrm{X}} \propto t^{-\beta}$, where $1.5<\beta<2.0$ (Güdel, Guinan \& Skinner 1997). Therefore, the late type companions of CSPNe should exhibit low levels of X-ray emission since a PN progenitor takes a considerable time to reach the AGB and PN phases.

Given these expectations, it is remarkable that the levels of X-ray emission in DS 1 , HFG 1, and LoTr 5, where we have identified late type companions, are high, at the saturated level. Take the example of LoTr 5. The X-ray emission comes from the G5 III companion which is known to rotate at a speed close to break-up, $v_{\text {rot }} \sin (i) \sim 67 \mathrm{~km} \mathrm{~s}^{-1}$ and display an extremely high $L_{\mathrm{X}} / L_{\text {bol }}$ ratio $\sim 10^{-3}$. Only such high $L_{\mathrm{X}} / L_{\mathrm{bol}}$ ratio is consistent with the G5 III spectral type of its companion (Fig. 3-right). Similar high levels of X-ray emission are observed in DS 1 and HFG 1 (Montez et al. 2010).

The abnormally fast rotation of the companion of LoTr 5 hints at the transfer of angular momentum from the PN progenitor to the companion. This hypothesis is also supported by the faster rotation of giants in symbiotic stars, as compared to field giant stars (Zamanov et al. 2006) and by the discovery of rapid-rotating companions of in the WD binary systems 2RE J0044+093 and 2RE J0357+283 (Jeffries \& Stevens 1996). The transfer of angular momentum may occur through the tidal synchronization in close binaries, or wind accretion in wide binaries (Jeffries \& Stevens 1996). A specific study 
for progenitors of PNe suggests that F7-M4 companions can be spun up to a period of 3 days (Soker \& Kastner 2002).

\subsection{Shock in winds}

The stellar winds of CSPNe are in many aspects similar to those of OB and Wolf-Rayet (WR) stars (Prinja 1990). These stellar winds are radiatively-driven, i.e., line scattering transfers energy and momentum from the stellar radiation to the material in the wind. The strong dependence of this driving mechanism with the material velocity makes it highly unstable, so that small-scale instabilities can lead to strong shocks within the dense innermost layers of the wind that generate gas at X-ray-emitting temperatures (Lucy \& White 1980; Gayley \& Owocki 1995).

The X-ray emission from the CSPNe of NGC 2392, NGC 6543, and NGC 6826 have plasma temperatures that are typical of single OB stars and whose X-ray to bolometric luminosity ratios are consistent with the canonical $\sim 10^{-7}$ value for OB stars (Berghöfer et al. 1997; Nazé 2009). Furthermore, the stellar winds of these CSPNe show evidence of variability of P Cygni profiles of UV high-excitation lines (Patriarchi \& Perinotto 1995, 1997; Guerrero \& de Marco 2012, in prep.) and even discrete absorption components (DACs) moving blue-wards (Prinja et al. 2007) that are similar to these seen in OB stars. The detection of $\mathrm{O}$ vi lines in the stellar winds of these stars is also compelling evidence of the occurrence of shocks within the stellar winds that heats the material up to X-ray-emitting temperatures (Herald \& Bianchi 2011).

\subsection{Colliding winds}

In OB or WR binary systems, the shock of the respective stellar winds can also produce shock-heated plasma. The X-ray emission from these systems is characterized by extremely hard X-ray emission, implying high plasma temperatures, $\sim 10^{7} \mathrm{~K}$, large hydrogen absorption column densities, and strong variability on orbital period time-scales, because the varying amount of un-shocked attenuating material along the line of sight (e.g., Gosset et al. 2009). The hard X-ray emission from CSPNe does not seem to fit these properties and thus colliding winds can be disregarded.

\subsection{Accretion}

Accretion of material onto a compact object (or an accretion disk around it) is one of the most powerful mechanisms able to produce hot plasma at X-ray-emitting temperatures in many different astrophysical scenarios. Note, however, that the strong stellar winds of NGC 6543 and NGC 6826 will inhibit the accretion of material onto the stellar surface. Therefore, accretion is not a viable mechanisms for these CSPNe.

The accretion of material from a mass transferring companion onto a WD or an accretion disk around it results in binary systems that are known as CVs (Cataclysmic variables). These systems emit strong UV and X-ray emission, but they have larger X-ray luminosities, hotter X-ray temperatures, and more variable X-ray light curves (Eracleous, Halpern \& Patterson 1991; Orio, Covington \& Ögelman 2001) than CSPNe. Furthermore, these systems are expected to show noticeable optical variability.

Material can also be accreted from the circumstellar medium. This has been suggested to be the origin of the hard X-ray emission from NGC 7293, being the result of intermittent accretion of material from a debris dust disk ( $\mathrm{Su}$ et al. 2007) as the observed level of X-ray emission cannot be produced by a putative companion that would be easily detected in the near- and mid-IR (Fig. 1-right). The accretion of material from a dust disk would also explain the variability of the $\mathrm{H} \alpha$ and [N II] stellar lines observed by Gruendl et al. (2001). However, the subtle variability of the hard X-ray emission from NGC 7293 
favors a constant accretion rate, rather than intermittent accretion (e.g., the plunging onto the surface of NGC 7293 CSPN of Kuiper-Belt-like objects) as it would result in large variability of the X-ray emission ( $\mathrm{Su}$ et al. 2007).

Finally, we note the suspicion that the Eskimo Nebula harbors a compact object at its core (Méndez et al. 2012). If this were the case, then the X-ray emission from its CSPN could be produced by the accretion of the CSPN stellar wind onto the surface of that compact object.

\subsection{Leakage of emission from deep shells in the stellar atmosphere}

The detection of hard X-ray emission from apparently single hot WDs by O'Dwyer et al. (2003) may imply a completely different mechanism for the production of hard X-ray emission in CSPNe. Up to date, single WDs with hard X-ray emission are rare (Bilíková et al. 2010). In all cases, these are very hot WDs with low metal content that makes their atmospheres very transparent to X-rays. This motivated the suggestion that X-rays originates from the leakage of emission from underneath the stellar atmosphere (Chu et al. 2004), although detail modeling has failed so far to reproduce this emission. As for the sample of CSPNe with hard X-ray emission, none of them seem to be capable of producing this emission, except for the recently discovered hard X-ray emission at the CSPN of K1-16 (Montez et al. 2012), because these CSPNe are colder than the hard X-ray-emitting WDs.

\section{Conclusions}

There is a growing number of CSPNe and WDs that display hard X-ray emission. This is not probably a single phenomenon, i.e., there is not a single mechanism responsible for the X-ray emission from all CSPNe.

Coronal emission from late-type companions is certainly a very appealing origin for the hard X-ray emission, as it would make X-ray observations useful to detect unseen companions. The X-ray observations of binary CSPNe indicate that the companions have enhanced coronal activity and rotation at late times in their evolution, suggesting the transfer of angular momentum.

Shocks within the stellar winds, as in OB stars, is also a viable mechanism for the production of hard X-rays, which is supported by the detection of super-ions such as O VI in relatively cool CSPNe and by evidence of wind variability. Other wind interactions, such as colliding winds, can be neglected.

Other mechanisms include leaking from underneath the atmosphere, as in hot WDs, and accretion. The former is unlikely, due to the lower surface temperature of the hard X-ray-emitting CSPNe, but accretion from circumstellar material may be occurring in NGC 7293. Accretion onto a compact companion is a tantalizing possibility for the CSPN of NGC 2392.

\section{Acknowledgements}

This work is mostly based on a long-term collaboration with Prof. You-Hua Chu and Dr. Robert A. Gruendl of the University of Illinois. I also acknowledge the support of grant AYA2008-01934 of the Spanish Ministerio de Ciencia e Innovación (MICINN) that includes FEDER funds.

\section{References}

Apparao, K. M. V. \& Tarafdar, S. P. 1989, ApJ, 344, 826 
Balick, B. 2004, AJ, 127, 2262

Berghöfer, T. W., Schmidtt, J. H. M. M., Danner, R., \& Cassinelli, J. P. 1997, A\&̋A, 322, 167

Bilíková, J., Chu, Y. H., Gruendl, R. A., \& Maddox, L. A. 2010, AJ, 140, 1433

Chu, Y. H., Guerrero, M. A., Gruendl, R. A., \& Webbink, R. F. 2004, AJ, 127, 477

Chu, Y. H., Guerrero, M. A., Gruendl, R. A., Williams, R. M., \& Kaler, J. B. 2001, ApJ, 553, L69

de Korte, P. A. J., Claas, J. J., Jansen, F. A., \& McKechnie, S. P. 1985, AdSpR, 5, 57

de Marco, O. 2009, PASP, 121, 316

Eracleous, M., Halpern, J., \& Patterson, J. 1991, ApJ, 382, 290

Fleming, T. A., Werner, K., \& Barstow, M. A. 1993, ApJ, 416, L79

Gayley, K. G. \& Owocki, S. P. 1995, ApJ, 446, 801

Gosset, E., Nazé, Y., Sana, H., Rauw, G., \& Vreux, J. M. 2009, A\&̊A, 508, 805

Gruendl, R. A., Chu, Y. H., O'Dwyer, I. J., \& Guerrero, M. A. 2001, AJ, 122, 308

Güdel, M. 2004, A\&̈A Rev. 12, 71

Güdel, M., Guinan, E. F., \& Skinner, S. L. 1997, ApJ, 483, 947

Guerrero, M. A., Chu, Y. H., \& Gruendl, R. A. 2000, ApJS, 129, 295

Guerrero, M. A., Chu, Y. H., Gruendl, R. A., Williams, R. M., \& Kaler, J. B. 2001, ApJ, 553, L55

Guerrero, M. A., Miranda, L. F., Riera, A., et al. 2008, ApJ, 683, 272

Herald, J. \& Bianchi, L. 2011, MNRAS, 417, 2440

Hoare, M. G., Martin, A. B., Werner, K., \& Fleming, T. 1995, MNRAS, 273, 812

Hünsch, M., Schmitt, J. H. M. M., Sterzik, M. F., \& Voges, W. 1999, A\&GAS, 135, 319

Hünsch, M., Schmitt, J. H. M. M., \& Voges, W. 1998, A\&SAS, 132, 155

Jasniewicz, G., Duquennoy, A., \& Acker, A. 1987, A\&SA, 180, 145

Jeffries R. D., Stevens I. R., 1996, MNRAS, 279, 180

Kreysing, H. C., Diesch, C., Zweigle, J., Staubert, R., Grewing, M., \& Hasinger, G. 1992, A\&A, 264,623

Leahy, D. A., Zhang, C. Y., \& Kwok, S. 1994, ApJ, 422, L205

Livio, M. \& Pringle, J. E. 1996, ApJ, 465, L55

Lucy, L. B. \& White, R. L. 1980, ApJ, 241, 300

Méndez, R., et al. 2012, IAU Symp. 283, this volume

Miranda, L. F. \& Solf, J. 1992, A $\& A$, 260, 397

Miszalski, B., Acker, A., Parker, Q. A., \& Moffat, A. F. J. 2009, A 8 A, 505, 249

Montez, R., Jr., De Marco, O., Kastner, J. H., \& Chu, Y. H. 2010, ApJ, 721, 1820

Montez, R., et al. 2012, IAU Symp. 283, this volume

Nazé, Y. 2009, A\& A, 506, 1055

O'Dwyer, I. J., Chu, Y. H., Gruendl, R. A., Guerrero, M. A., \& Webbink, R. F. 2003, AJ, 125, 2239

Orio, M., Covington, J., \& Ögelman, H. 2001, A\&A, 373, 542

Pallavicini, R., Golub, L., Rosner, R., Vaiana, G. S., Ayres, T., \& Linsky, J. L. 1981, ApJ, 248, 279

Patriarchi, P. \& Perinotto, M. 1995, A\&AS, 110, 353

Patriarchi, P. \& Perinotto, M. 1997, A\&AS, 126, 385

Prinja, R. K. 1990, A\& A, 232, 119

Prinja, R. K., Hodges, S. E., Massa, D. L., Fullerton, A. W., \& Burnley, A. W. 2007, MNRAS, 382,299

Rauch, T. 2003, A\&SA, 403, 709

Rauch, T., Köppen, J., \& Werner, K. 1994, A\&AA, 286, 543

Soker, N. \& Kastner, J. H. 2002, ApJ, 570, 245

Soker, N. 1997, ApJS, 112, 487

Su, K. Y. L., Chu, Y. H., Rieke, G. H., Huggins, P. J., Gruendl, R. A., Napiwotzki, R., Rauch, T., Latter, W. B., \& Volk, K. 2007, ApJ, 657, L41

Tarafdar, S. P. \& Apparao, K. M. V. 1988, ApJ, 327, 342

Zamanov, R. K., Bode, M. F., Melo, C. H. F., Porter, J., Gomboc, A., \& Konstantinova-Antova, R. 2006, MNRAS, 365, 1215 